\title{
Baseline characteristics predict risk of progression and response to combined medical therapy for benign prostatic hyperplasia (BPH)
}

\author{
Michael A. Kozminski, John T. Wei, Jason Nelson* and David M. Kent* \\ Department of Urology, University of Michigan Medical School, Ann Arbor, MI, and *Predictive Analytics and \\ Comparative Effectiveness Center, Institute for Clinical Research and Health Policy Studies, Tufts Medical Center/Tufts \\ University School of Medicine, Boston, USA
}

\section{Objective}

To better risk stratify patients, using baseline characteristics, to help optimise decision-making for men with moderate-tosevere lower urinary tract symptoms (LUTS) secondary to benign prostatic hyperplasia (BPH) through a secondary analysis of the Medical Therapy of Prostatic Symptoms (MTOPS) trial.

\section{Patients and Methods}

After review of the literature, we identified potential baseline risk factors for $\mathrm{BPH}$ progression. Using bivariate tests in a secondary analysis of MTOPS data, we determined which variables retained prognostic significance. We then used these factors in Cox proportional hazard modelling to: i) more comprehensively risk stratify the study population based on pre-treatment parameters and ii) to determine which risk strata stood to benefit most from medical intervention.

\section{Results}

In all, 3047 men were followed in MTOPS for a mean of 4.5 years. We found varying risks of progression across quartiles. Baseline BPH Impact Index score, post-void residual urine volume, serum prostate-specific antigen (PSA) level, age, American Urological Association Symptom Index score, and maximum urinary flow rate were found to significantly correlate with overall $\mathrm{BPH}$ progression in multivariable analysis.

\section{Conclusions}

Using baseline factors permits estimation of individual patient risk for clinical progression and the benefits of medical therapy. A novel clinical decision tool based on these analyses will allow clinicians to weigh patient-specific benefits against possible risks of adverse effects for a given patient.

\section{Keywords}

benign prostatic hyperplasia $(\mathrm{BPH})$, lower urinary tract symptoms (LUTS), risk factors

\section{Introduction}

$\mathrm{BPH}$ can progress over time. Community based studies have estimated that $>30 \%$ of men will experience clinical progression in the course of 5 years [1-4], predominantly characterised by increases in LUTS severity $[5,6]$. While BPH is highly prevalent, not all men with BPH have the same risk for progression $[7,8]$.

Determining predictors of this risk has been an ongoing process over the past decade. A few trials have examined their placebo arms to elucidate baseline characteristics that portend worse prognoses $[9,10]$. However, these efforts have generally focused on a single factor, such as initiation of $5 \alpha$-reductase inhibitors (5-ARIs), for men with enlarged prostates or elevated PSA levels. In clinical practice, this may translate into focusing on an isolated variable to risk stratify when patients have multiple factors that might simultaneously influence the risk of progression and the potential benefits of therapy.

With the combined use of $\alpha$-blockers and 5-ARIs, men can reduce their risk of $\mathrm{BPH}$ progression and ultimate need for invasive procedures. The Medical Therapy of Prostatic Symptoms (MTOPS) trial demonstrated clear benefit in risk reduction with this medical combination [6]. Classically, clinical trials showing a positive result suggest that practitioners should adopt a 'treat all' strategy. Yet, just as patient risk of progression can be variable, a given patient's response to medical therapy may diverge from the mean, a concept known as heterogeneity of treatment effect.

It has recently been proposed that the results of clinical trials be routinely analysed and presented in a risk-stratified fashion 
to examine the relative and absolute effects across different risk strata, as baseline risk is a mathematical determinant of the treatment effect and can differ greatly across patients in a trial [11-13]. To date, it remains unknown how the benefits of available therapies for BPH vary across patients at different progression risks, and such information could have important implications for clinical practice.

To better aid clinicians with decision making, we sought to risk stratify men with moderate-to-severe LUTS secondary to $\mathrm{BPH}$ using established risk factors in a data-driven model. With data from the MTOPS, we examined trial outcomes across risk strata of $\mathrm{BPH}$ progression to better define which patients are most likely to benefit from $\alpha$-blockers, 5-ARIs, or their combination.

\section{Patients and Methods}

We obtained original, publicly available data from the MTOPS study. The MTOPS study was conducted by the MTOPS Investigators and supported by the National Institute of Diabetes and Digestive and Kidney Diseases (NIDDK). Approval for our present analysis was provided by NIDDK and the study was approved by the Tufts Medical Center Internal Review Board [14]. The design, rationale, and outcomes of the MTOPS study are described in detail elsewhere [6]. In short, MTOPS was a randomised trial evaluating doxazosin, finasteride, or the combination of these medications for risk of $\mathrm{BPH}$ progression in men aged $\geq 50$ years with AUA Symptom Index (AUA-SI) scores of 8-30 and maximum urinary flow rates $\left(\mathrm{Q}_{\max }\right)$ of $4-15 \mathrm{~mL} / \mathrm{s}$ enrolled between 1993 and 1998. BPH progression, or the primary outcome, was defined as 'the first occurrence of an increase over base line of at least four points in the AUA-SI score, acute urinary retention, renal insufficiency, recurrent urinary tract infection, or urinary incontinence' [6].

To capture potential variables for risk stratification, we reviewed the current literature. Within their primary analysis, MTOPS investigators found that baseline PSA level and baseline prostate volume significantly correlated with progression in univariate analysis [6]. We identified two other models predictive of BPH progression based on: i) randomised-controlled trials with a different 5-ARI (dutasteride) $[8,15]$ and ii) expert consensus [16]. These revealed additional potential risk factors: age, severe symptoms (as defined by both the BPH Impact Index (BII) [17] and the AUA-SI [18]; Appendix 1), lower $\mathrm{Q}_{\max }$, and elevated post-void residual urine volume (PVR). Review of other community based surveys and placebo-controlled trials further supported the relevance of these variables $[1,2,19-23]$.

Using Cox proportional hazards regression, we conducted univariate tests to determine which of these variables, measured at baseline, were associated with the outcome. We then used the factors with $P$-values $<0.1$ in a multivariate Cox model, excluding treatment assignment. As the GP does not typically assess PVR or $\mathrm{Q}_{\max }$ in a primary care setting, we attempted to make our work more broadly applicable by creating a reduced model with easily measured factors (age, PSA level, AUA-SI, BII). The full and reduced Cox models were internally validated with bootstrapping to quantify optimism in model performance. We evaluated model performance with the c-statistic [24]. Calibration was evaluated with a calibration plot and modified Hosmer-Lemeshow chi-squared test for survival analysis [25] and was excellent (chi-squared $P=0.99$ ).

From both the full and reduced Cox regression models, in separate analyses, we stratified the trial population into equal-sized risk quartiles. Within each risk quartile, we calculated the active drug treatment effect compared with placebo for each of the three intervention arms (i.e., doxazosin, finasteride, combination). Using Kaplan-Meier estimated failure rates after 4 years of follow-up, we calculated the absolute risk reduction and number needed to treat for each drug or drug combination compared with placebo, as well as the combined therapy compared with each active drug individually. Heterogeneity of treatment effect was assessed by including an interaction term in the Cox regression model between the baseline linear risk predictor and treatment assignment to estimate the effect of treatment as a function of risk in $\mathrm{BPH}$ progression.

Finally, with results from the internally validated models, we constructed a clinical decision tool (nomogram) for care providers. Data management and regression model building were performed using SAS version 9.3 (SAS Institute, Cary NC). We used Frank Harrell's 'rms' package in R software version 3.0.1 to perform the bootstrapped internal validation and generate the nomogram.

\section{Results}

In all, 3047 men were followed in MTOPS for a mean of 4.5 years. They were randomised to four arms: placebo (737 men), doxazosin (756), finasteride (768), and combined therapy (786). In all, 351 primary outcome events (i.e., BPH progression events) occurred in total, with 128 in the placebo arm, 85 in the doxazosin arm, 89 in the finasteride arm, and 49 in the combination arm. These were predominantly characterised as increases in symptom severity (78\%), but did include acute urinary retention (12\%), incontinence (9\%), and recurrent UTI/urosepsis in five cases. The 4-year overall risk of clinical progression within the placebo arm was $17 \%$, compared with $10 \%$ in the doxazosin arm, $10 \%$ in finasteride arm, and 5\% in combined therapy arm (all previously reported $P$-values $<0.002$ ) [6]. Baseline characteristics of the study patients are shown in Table 1. Because collection of the variables of interest was excellent, we used complete case 
Table 1 Baseline characteristics of MTOPS cohort.

\begin{tabular}{|c|c|c|c|c|c|}
\hline Characteristic & Cohort & Placebo & Doxazosin & Finasteride & Combined therapy \\
\hline Number of patients & 3047 & 737 & 756 & 768 & 786 \\
\hline \multicolumn{6}{|l|}{ Mean (SD) } \\
\hline Age, years (range 50-89) & $62.6(7.3)$ & $62.5(7.6)$ & $62.7(7.3)$ & $62.6(7.3)$ & $62.7(7.1)$ \\
\hline AUA-SI score (range 8-35) & $16.9(5.9)$ & $16.8(6.0)$ & $17(5.9)$ & $17.1(6.0)$ & $16.8(5.8)$ \\
\hline Prostate volume, $\mathrm{mL}$ (range 6.1-185.0) & $36.3(20.1)$ & $35.2(18.9)$ & $36.9(21.6)$ & $36.9(20.6)$ & $36.4(19.2)$ \\
\hline $\mathrm{Q}_{\max }, \mathrm{mL} / \mathrm{s}$ (range 4-15.3) & $10.5(2.6)$ & $10.5(2.7)$ & $10.3(2.6)$ & $10.5(2.6)$ & $10.6(2.5)$ \\
\hline PVR, mL (range 0-789) & $68.1(82.9)$ & $69.6(82.1)$ & $69.2(88.3)$ & $66.2(80.1)$ & $67.5(81.2)$ \\
\hline Serum PSA level, ng/mL (range 0.2-10.5) & $2.4(2.1)$ & $2.3(2.1)$ & $2.4(2.2)$ & $2.4(2.1)$ & $2.3(2.0)$ \\
\hline Serum glucose level, ng/dL (range 6-465) & $100.1(42.5)$ & $100.8(43.9)$ & $99.3(41.6)$ & $98.6(37.4)$ & $101.6(46.4)$ \\
\hline Body mass index, $\mathrm{kg} / \mathrm{m}^{2}$ (range $16.5-52.1$ ) & $27.8(4.2)$ & $27.6(4.0)$ & $27.7(3.9)$ & $27.9(4.6)$ & $27.9(4.2)$ \\
\hline BII score (range 0-13) & $4.0(2.7)$ & $4.0(2.7)$ & $3.9(2.7)$ & $4.1(2.8)$ & $4.0(2.7)$ \\
\hline \multicolumn{6}{|l|}{$N(\%)$} \\
\hline Primary school education only & $120(3.9)$ & $29(3.9)$ & $36(4.8)$ & $31(4.0)$ & $24(3.1)$ \\
\hline \multicolumn{6}{|l|}{ Family history of prostate cancer: } \\
\hline Yes & $416(13.7)$ & $95(12.9)$ & $94(12.4)$ & $98(12.8)$ & $129(16.4)$ \\
\hline No & $2228(73.1)$ & $545(74.0)$ & $546(72.2)$ & $578(75.3)$ & $559(71.1)$ \\
\hline Unknown & $403(13.2)$ & $97(13.2)$ & $116(15.3)$ & $92(12.0)$ & $98(12.5)$ \\
\hline \multicolumn{6}{|l|}{ Erectile dysfunction: } \\
\hline Yes & $747(24.6)$ & $185(25.1)$ & $196(26.0)$ & $184(24.0)$ & $182(23.2)$ \\
\hline No & $1745(57.4)$ & $417(56.7)$ & $423(56.0)$ & $456(59.4)$ & $449(57.3)$ \\
\hline Intermittent & $550(18.1)$ & $134(18.2)$ & $136(18.0)$ & $128(16.7)$ & $152(19.4)$ \\
\hline History of diabetes & $260(8.5)$ & $72(9.8)$ & $59(7.8)$ & $65(8.5)$ & $64(8.1)$ \\
\hline History of hypertension & $871(28.6)$ & $203(27.5)$ & $226(29.9)$ & $212(27.6)$ & $230(29.3)$ \\
\hline
\end{tabular}

HR (95\% CI) for the treatment arms were as follows: doxazosin 0.54 (0.40-0.72), finasteride 0.60 (0.45-0.80), combination 0.27 (0.19-0.39).

Table 2 Bivariate analysis: baseline characteristics and their correlation with risk of BPH progression Characteristics identified within our literature search, as well as a number of other factors collected within MTOPS data were analysed. Variables are plotted with their model coefficients and HRs. Standard errors are noted for each estimate.

\begin{tabular}{lrrrr}
\hline Variable & Estimate & SE & $\boldsymbol{P}$ & HR \\
\hline Age, year & 0.037 & 0.007 & $<0.001$ & 1.038 \\
AUA-SI score & -0.024 & 0.009 & 0.010 & 0.976 \\
Prostate volume, $\mathrm{mL}$ & 0.010 & 0.002 & $<0.001$ & 1.010 \\
$\mathrm{Q}_{\max } \mathrm{mL} / \mathrm{s}$ & -0.059 & 0.020 & 0.004 & 0.943 \\
PVR, mL & 0.002 & 0.001 & 0.001 & 1.002 \\
Serum PSA level, ng/mL & 0.110 & 0.022 & $<.001$ & 1.116 \\
BII score & 0.045 & 0.019 & 0.018 & 1.046 \\
Serum glucose, ng/dL & -0.001 & 0.001 & 0.713 & 1.000 \\
Body mass index, kg/m ${ }^{2}$ & -0.002 & 0.013 & 0.893 & 0.998 \\
Primary school education only, yes vs no & 0.351 & 0.242 & 0.147 & 1.421 \\
Family history of prostate cancer & & & & 0.927 \\
$\quad$ Yes & -0.076 & 0.160 & 0.636 & 1.00 (reference) \\
$\quad$ No & & & & 1.226 \\
$\quad$ Unknown & 0.204 & 0.148 & 0.168 & 1.236 \\
Erectile dysfunction & & & & 1.00 (reference) \\
$\quad$ Yes & 0.212 & 0.119 & 0.076 & 1.007 \\
$\quad$ No & & & & 1.086 \\
$\quad$ Intermittent & 0.007 & 0.137 & 0.958 & 1.017 \\
History of diabetes, yes vs no & 0.083 & 0.185 & 0.656 & 0.884 \\
History of hypertension, yes vs no & 0.017 & 0.119 & & \\
\hline
\end{tabular}

analysis and excluded only nine subjects from the full multivariate model and four from the reduced multivariate model.

Bivariate analysis was performed on all clinically relevant variables based on our literature review (Table 2). Variables showing statistical significance were then used to create a parsimonious, multivariable model of $\mathrm{BPH}$ progression risk.
Baseline BII score (per 1 point, hazard ratio [HR] 1.12, 95\% CI 1.07-1.17), PVR (per $100 \mathrm{~mL}, \mathrm{HR} 1.17,95 \%$ CI 1.04-1.30), serum PSA level (per $5 \mathrm{ng} / \mathrm{mL}$, HR 1.44, 95\% CI 1.14-1.82), age (per 10 years, HR 1.33, 95\% CI 1.15-1.53), AUA-SI score (per 5 points, HR $0.76,95 \%$ CI $0.68-0.85$ ), and $\mathrm{Q}_{\max }$ (per $5 \mathrm{~mL} / \mathrm{s}$, HR $0.74,95 \%$ CI $0.60-0.90$ ) were found to be significantly negatively correlated with overall $\mathrm{BPH}$ progression in multivariable analysis (Table 3 ). Internal 
validation after 500 bootstrap repetitions revealed an optimism-corrected c-statistic of 0.626 compared with 0.635 in the original dataset.

This predictive model of risk for BPH progression (c-statistic $=0.635$ ) showed varying risks of progression across quartiles (Fig. 1). Formal tests of interaction between risk and treatment were not significant (data not shown), indicating that patients experienced roughly proportional benefits across risk strata from the three different treatments. However, the highest-risk quartile had a risk of progression $\approx 300 \%$ that of the lowest within all trial arms on the absolute risk reduction scale. Thus, the number needed to treat for those patients in the lowest quartile of risk was about three-times that for patients in the highest quartile. MTOPS showed a $17 \%$ overall incidence of clinical progression for the 'average' patient [6], but a patient within the lowest quartile only bears a progression risk of $10.7 \%$, while the typical highest quartile patient faces up to a $29.6 \%$ risk of progression. To facilitate a more direct comparison between serious adverse events (SAEs) and

Table 3 Multivariable Cox proportional hazards model. Variables are listed with their respective HRs. Prostate volume did not (by neither DRE nor TRUS estimate) maintain significance in our multivariable model.

\section{Variable} HR

Age (per 10 years)
Serum PSA (per $5 \mathrm{ng} / \mathrm{mL}$ )
AUA-SI score (per 5 points)
BII score (per 1 point)
Qmax (per $5 \mathrm{~mL} / \mathrm{s}$ )
PVR (per $100 \mathrm{~mL}$ )

$\mathrm{N}=3036$ with non-missing data for all predictors. $C L$, confidence limit. $c$-statistic $=$ 0.635 . therapeutic benefits, we stratified those SAEs that resulted in medication interruption or discontinuation that occurred before a primary outcome event within the first 4 years of follow-up (Fig. 2). This figure shows a clear increase in the ratio of the benefits to the side-effects of therapy as risk increases.

Our reduced model retained predictive value with a c-statistic of 0.623 (Table 4), compared with 0.635 with the full model (Table 3). This suggests that these easily attainable parameters can be used in various clinical settings to predict risk of $\mathrm{BPH}$ progression. Clinical decision tools were then constructed from these models (Appendix 2 and Fig. 3).

\section{Discussion}

While MTOPS reported an overall incidence of clinical progression of $17 \%$ in the placebo group, we showed that this risk is variable among men. Whereas benefits of medical therapy for patients with a high risk of progression are clear, the benefits to patients at lower risk are less so, and may be more finely balanced with risks and sensitive to patient preferences.

Prior studies have offered several predictive baseline factors for the clinical progression of BPH. These have included increased symptom severity (both AUA-SI and BII), low $\mathrm{Q}_{\max }$, high PVR, high prostate volume, and high serum PSA level $[8,9,19,20,26]$. Age has also been reported to have prognostic value [6]. Similar to these previous reports, we found several predictive characteristics, including age, serum PSA level, PVR, $Q_{\max }$, BII score, and AUA-SI score.

Of note, the AUA-SI score has a HR $<1$ in our model, indicating that higher symptoms are protective against progression. This is probably because those men with severe
Fig. 1 The 4-year risk of BPH progression stratified across quartiles. Linear predictor scores from our full model were used to generate a spectrum of risk, which we divided into quartiles. This was performed for all four arms of MTOPS. The observed mean event rates for each arm within each quartile were collected based on Kaplan-Meier estimated event rates within the MTOPS trial.

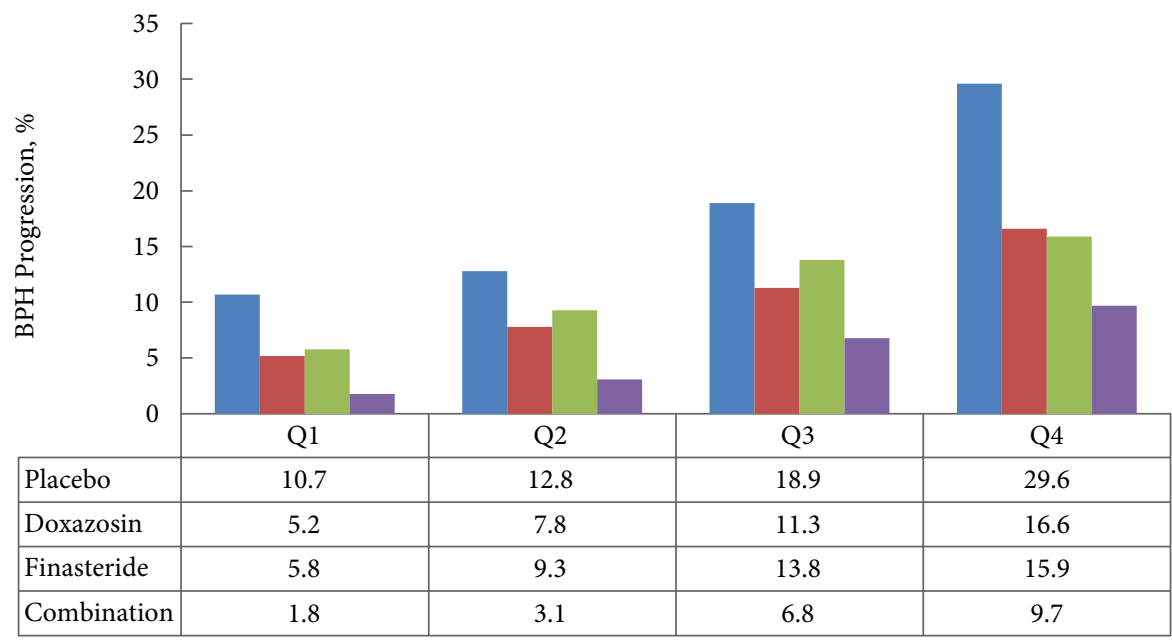

Quartiles (based on risk of BPH progression in MTOPS)

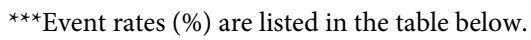




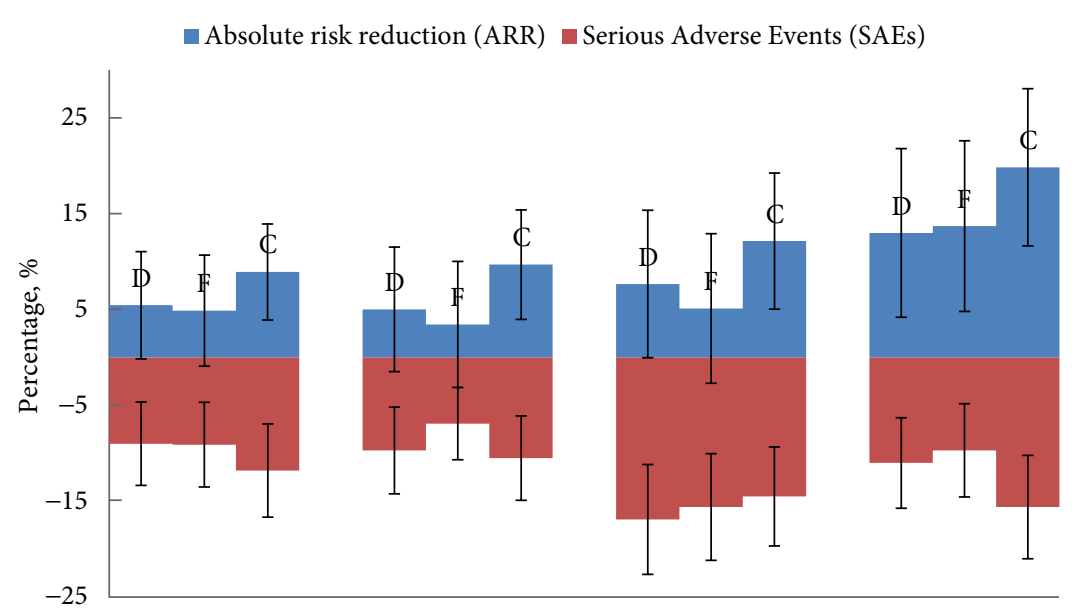

Fig. 2 Medication treatment effects in terms of absolute risk compared to SAEs that resulted in therapy interruption/discontinuation. Absolute risk reductions (with $95 \% \mathrm{Cl}$ ) for the different treatment arms are calculated compared with the placebo arm and increase over quartiles of BPH progression, ordered from left to right (Q1->Q4), with each treatment arm reported (ordered as doxazosin (D), finasteride (F), combination (C) in each quartile). SAEs (with 95\% $\mathrm{Cl}$ ) are reported in raw percentages (listed as negative values for comparison). The number needed to treat (NNT) is derived from the absolute risk reductions in the respective groups, as listed in the table below.

\begin{tabular}{|l|c|c|c|c|}
\hline NNT (vs placebo) & Q1 & Q2 & Q3 & Q4 \\
\hline Doxazosin & 19 & 20 & 14 & 7 \\
\hline Finasteride & 21 & 30 & 20 & 8 \\
\hline Combination & 12 & 11 & 9 & 6 \\
\hline vs doxazosin & 30 & 22 & 23 & 15 \\
\hline vs finasteride & 25 & 17 & 15 & 17 \\
\hline
\end{tabular}

ARR, absolute risk reduction compared to placebo; NNT, number needed to treat (1/ARR).

Table 4 Reduced multivariable Cox proportional hazards model. This model retains its predictive value over a similar range of decision thresholds compared with the full model (Table 3). However, this model includes variables that may be easily measured in primary care settings allowing for broader applicability.

\section{Variable} HR

Age (per 10 years)

Serum PSA (per $5 \mathrm{ng} / \mathrm{mL}$ )

AUA-SI score (per 5 points)

BII score (per 1 point)

$\mathrm{N}=3043$ with non-missing data for all predictors. $C L$, confidence limit. $c$-statistic $=$ 0.623 .

symptoms were less likely to progress to even worse symptoms (as detected by the AUA-SI) during the follow-up period.

In our final model, prostate volume was not necessary to yield optimal prognostic information. While seemingly in contrast to prior studies $[6,10,26,27]$, which suggested that larger prostate volumes are associated with higher risk of $\mathrm{BPH}$ progression, our multivariable analysis suggests that once PSA level and symptom severity score are included, the information gained from knowing prostate size was no longer statistically significant in predicting $\mathrm{BPH}$ progression. This finding is particularly relevant in the primary care setting, where the availability of prostate ultrasound to measure size is uncommon. Of note, our findings cannot be generalised to men outside the MTOPS entry criteria.
Prior analyses $[6,27,28]$ suggested that elevated serum PSA level and higher total prostate volume were the strongest predictors of $\mathrm{BPH}$ progression. The present analysis suggests that focusing on these two factors in isolation would fail to capture the entire clinical picture for a given patient. As one can imagine, in patients who have conflicting characteristics, decisions made on a single factor may inappropriately guide therapy. Moreover, univariable analyses tend to highlight a certain threshold (e.g., a patient's prostate is considered enlarged if it is $>30 \mathrm{~mL}$ ), but such decision points can arbitrarily endorse treatment or no treatment again for the non-ideal candidate.

Using our present derived nomogram will allow clinicians to account for the key factors that have been shown to predict disease progression in a large, prospective clinical trial. A patient's age, AUA-SI score, BII score, and serum PSA level can easily be collected during a clinical encounter. These values can then be translated into a 'Total Points' within our nomogram, as described in Fig. 3, and ultimately into a risk of progression. Clinicians can then weigh this risk of progression against the potential risk of side-effects, as briefly outlined in Fig. 2, and other patient-specific characteristics (e.g., patient compliance, medication costs to the patient). We hope to ultimately translate this nomogram into a user-friendly, web-based application.

Other groups have created clinical tools in an effort to help physicians most effectively employ medical therapy for $\mathrm{BPH}$. Lowe et al. [16] surveyed 12 international 'experts' on $\mathrm{BPH}$ using over 240 hypothetical patient scenarios to determine the 
Fig. 3 Reduced model. Clinical decision tool for predicting 4-year risk of clinical BPH

Points progression using data from MTOPS, four predictor regression model. To determine an individual's risk of progression, draw a vertical line from each of the patient's factors to the 'Points' line above. Add the four 'Points' values together to arrive at a 'Total Points'. Calculate the expected event rate with therapy by applying the HRs below.
Age, years

AUA Symptom Score

BPH Impact Index

PSA, $\mathrm{ng} / \mathrm{mL}$



$\begin{array}{lllllllll}50 & 55 & 60 & 65 & 70 & 75 & 80 & 85 & 90\end{array}$

$\begin{array}{lllllllllllllll}36 & 34 & 32 & 30 & 28 & 26 & 24 & 22 & 20 & 18 & 16 & 14 & 12 & 10 & 8\end{array}$

$\begin{array}{llllllllllllll}0 & 1 & 2 & 3 & 4 & 5 & 6 & 7 & 8 & 9 & 10 & 11 & 12 & 13\end{array}$

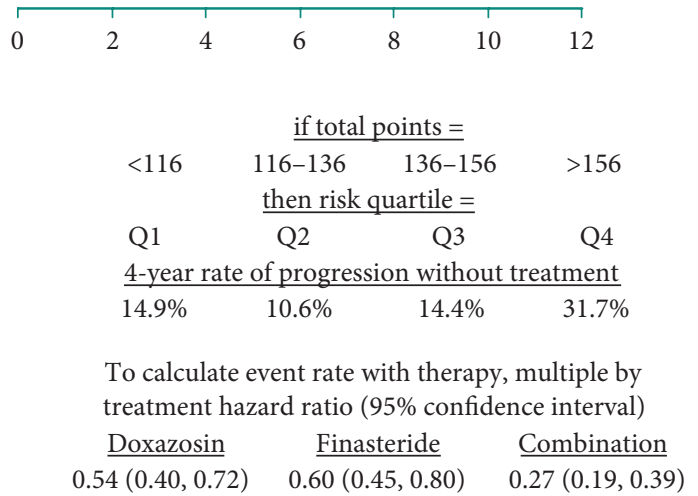

most important prognostic factors for progression. This panel concluded that symptom severity, low $\mathrm{Q}_{\max }$, and elevated PVR were the most important determinants for progression, although PSA level and prostate volume were also considered significant. The authors formulated a regression model to predict risk based on the panel's responses.

Additionally, Slawin et al. $[8,15]$ performed secondary analysis on three 2-year, multicentre, placebo-controlled, double-blind randomised trials with dutasteride to create a clinical nomogram for the risk of acute urinary retention or $\mathrm{BPH}$-related surgery. However, those data were limited to men with larger prostates $(>30 \mathrm{~mL})$ and had a shorter follow-up time than MTOPS (2 years vs 4.5 years). Moreover, MTOPS showed, in a more inclusive sample, that progression predominantly manifests as worsening symptom severity as opposed to retention or surgery. As such, the Slawin model can assist in more specialised, second-line settings, while our present model may be more widely applicable to the treatment-naïve population. Additionally, while they examined the variable risk of progression, ours remains the first study to examine combined medical therapy in a risk-stratified analysis.

Clinical decisions must also consider the AEs of possible interventions. For those men in MTOPS who received active therapy, 27\% stopped doxazosin, 24\% stopped finasteride, and $18 \%$ stopped combined therapy. Medication cessation was most often because of AEs [6]. We found that the risk of medication discontinuation was relatively stable across quartiles of progression risk, at 8-15\%. Clinicians can use our present decision tool to weigh a patient's risk of progression against this risk of discontinuation to determine if there may be a realised benefit in initiating medical therapy.

As with most statistical models, our present findings may be subject to over-fitting and would benefit from external validation in another cohort. However, by basing our model on just a handful of clinically relevant variables and by having a large number of outcome events for each variable tested, over-fitting is less likely to have impacted our primary conclusions, although miscalibration to the external population could affect the benefits of using the model. Additionally, for methodological reasons, we excluded therapy from our model, which lowered the c-statistic; inclusion would have greatly improved discrimination, as the medications were so effective. Furthermore, while we postulated that our findings might improve effectiveness of these pharmacological interventions at the population level, no cost data were collected in the MTOPS trial, so cost-effectiveness analyses were not performed here.

In conclusion, the risk of $\mathrm{BPH}$ progression is highly variable among men. MTOPS data suggests that the benefits of medical therapy for $\mathrm{BPH}$ are unevenly distributed with men who were in the highest risk strata accounting for the greatest clinical 
benefit. Importantly, using commonly available baseline risk factors permits estimation of the patient-specific risk for clinical progression, and thus the potential for benefit. Our present novel decision tool based on clinically available factors (age, AUA-SI score, BII score, serum PSA level) may allow clinicians to better select those most likely to benefit from medical therapy for $\mathrm{BPH}$ and potentially inform future guidelines. Potential treatment effect can then be weighed against possible risks of AEs for a given patient.

\section{Acknowledgements}

J.N and D.M.K. had full access to all of the data in the study and take responsibility for the integrity of the data and the accuracy of the data analysis.

This manuscript was partially funded by grants $1 \mathrm{UL} 1$ TR001064 and U01 AA022802, both from the National Institutes of Health (NIH), as well as grant 1IP2PI000722, from the Patient-Centered Outcome Research Institute (PCORI).

The MTOPS study was conducted by the MTOPS Investigators and supported by the NIDDK. The data and samples from MTOPS reported here were supplied by the NIDDK Central Repositories. This manuscript was not prepared in collaboration with Investigators of the MTOPS study and does not necessarily reflect the opinions or views of the MTOPS study, the NIDDK Central Repositories, or the NIDDK.

\section{Conflicts of Interest}

None of the four authors have any conflicts of interest.

\section{References}

1 Flanigan RC, Reda DJ, Wasson JH, Anderson RJ, Abdellatif M, Bruskewitz RC. 5-year outcome of surgical resection and watchful waiting for men with moderately symptomatic benign prostatic hyperplasia: a Department of Veterans Affairs cooperative study. J Urol 1998; 160: 12-7

2 Jacobsen SJ, Girman CJ, Lieber MM. Natural history of benign prostatic hyperplasia. Urology 2001; 58 (Suppl. 1): 5-16

3 Maserejian NN, Chen S, Chiu GR et al. Treatment status and progression or regression of lower urinary tract symptoms in a general adult population sample. J Urol 2014; 191: 107-13

4 Martin S, Lange K, Haren MT et al. Risk factors for progression or improvement of lower urinary tract symptoms in a prospective cohort of men. J Urol 2014; 191: 130-7

5 Roehrborn CG. BPH progression: concept and key learning from MTOPS, ALTESS, COMBAT, and ALF-ONE. BJU Int 2008; 101 (Suppl. 3): $17-21$

6 McConnell JD, Roehrborn CG, Bautista OM et al. The long-term effect of doxazosin, finasteride, and combination therapy on the clinical progression of benign prostatic hyperplasia. N Engl J Med 2003; 349 : 2387-98

7 Anderson JB, Roehrborn CG, Schalken JA, Emberton M. The progression of benign prostatic hyperplasia: examining the evidence and determining the risk. Eur Urol 2001; 39: 390-9
8 Slawin KM, Kattan MW. The use of nomograms for selecting BPH candidates for dutasteride therapy. Rev Urol 2004; 6 (Suppl. 9): S40-45

9 Crawford ED, Wilson SS, McConnell JD et al. Baseline factors as predictors of clinical progression of benign prostatic hyperplasia in men treated with placebo. J Urol 2006; 175: 1422-7

10 Roehrborn CG, Siami P, Barkin J et al. The influence of baseline parameters on changes in international prostate symptom score with dutasteride, tamsulosin, and combination therapy among men with symptomatic benign prostatic hyperplasia and an enlarged prostate: 2-year data from the CombAT study. Eur Urol 2009; 55: 461-71

11 Kent DM, Rothwell PM, Ioannidis JP, Altman DG, Hayward RA. Assessing and reporting heterogeneity in treatment effects in clinical trials: a proposal. Trials 2010; 11: 85

12 Dorresteijn JA, Visseren FL, Ridker PM et al. Estimating treatment effects for individual patients based on the results of randomised clinical trials. BMJ 2011; 343: d5888

13 Burke JF, Hayward RA, Nelson JP, Kent DM. Using internally developed risk models to assess heterogeneity in treatment effects in clinical trials. Circ Cardiovasc Qual Outcomes 2014; 7: 163-9

14 Cuticchia AJ, Cooley PC, Hall RD, Qin Y. NIDDK data repository: a central collection of clinical trial data. BMC Med Inform Decis Mak 2006; 6: 19

15 Slawin KM, Kattan MW, Roehrborn CG, Wilson T. Development of nomogram to predict acute urinary retention or surgical intervention, with or without dutasteride therapy, in men with benign prostatic hyperplasia. Urology 2006; 67: 84-8

16 Lowe FC, Batista J, Berges R et al. Risk factors for disease progression in patients with lower urinary tract symptoms/benign prostatic hyperplasia (LUTS/BPH): a systematic analysis of expert opinion. Prostate Cancer Prostatic Dis 2005; 8: 206-9

17 Barry MJ, Fowler FJ, Jr, O’Leary MP, Bruskewitz RC, Holtgrewe HL, Mebust WK. Measuring disease-specific health status in men with benign prostatic hyperplasia. Measurement Committee of The American Urological Association. Med Care 1995; 33 (Suppl).):AS145-155

18 Barry MJ, Fowler FJ Jr, O'Leary MP et al. The American Urological Association symptom index for benign prostatic hyperplasia. The Measurement Committee of the American Urological Association. J Urol 1992; 148: 1549-57; discussion 1564

19 Roehrborn CG. Definition of at-risk patients: baseline variables. BJU Int 2006; 97 (Suppl. 2): 7-11; discussion 21-12

20 Trachtenberg J. Treatment of lower urinary tract symptoms suggestive of benign prostatic hyperplasia in relation to the patient's risk profile for progression. BJU Int 2005; 95 (Suppl. 4): 6-11

21 Hong SJ, Ko WJ, Kim SI, Chung BH. Identification of baseline clinical factors which predict medical treatment failure of benign prostatic hyperplasia: an observational cohort study. Eur Urol 2003; 44: 94-100

22 Kok ET, Schouten BW, Bohnen AM et al. Risk factors for lower urinary tract symptoms suggestive of benign prostatic hyperplasia in a community based population of healthy aging men: the Krimpen Study. J Urol 2009; 181: 710-6

23 Parsons JK. Modifiable risk factors for benign prostatic hyperplasia and lower urinary tract symptoms: new approaches to old problems. J Urol 2007; 178: 395-401

24 Steyerberg EW, SpringerLink. Clinical Prediction Models A Practical Approach to Development, Validation, and Updating. New York, NY: Springer-Verlag New York, 2009

25 D'Agostino R, Nam B. Evaluation of the Performance of Survival Analysis Models: Discrimination and Calibration Measures. Amsterdam, The Netherlands: Elsevier, 2004

26 Emberton M, Zinner N, Michel MC, Gittelman M, Chung MK, Madersbacher S. Managing the progression of lower urinary tract 
symptoms/benign prostatic hyperplasia: therapeutic options for the man at risk. BJU Int 2007; 100: 249-53

27 Kaplan SA, McConnell JD, Roehrborn CG et al. Combination therapy with doxazosin and finasteride for benign prostatic hyperplasia in patients with lower urinary tract symptoms and a baseline total prostate volume of $25 \mathrm{ml}$ or greater. J Urol 2006; 175: 217-21

28 Roehrborn CG, McConnell JD, Lieber M et al. Serum prostate-specific antigen concentration is a powerful predictor of acute urinary retention and need for surgery in men with clinical benign prostatic hyperplasia. PLESS Study Group. Urology 1999; 53: 473-80

Correspondence: David M. Kent, Predictive Analytics and Comparative Effectiveness (PACE) Center, Institute of Clinical
Research and Health Policy Studies, Tufts Medical Center, 800 Washington Street, Box 63, Boston, MA 02111, USA.

e-mail: dkent1@tuftsmedicalcenter.org

Abbreviations: (S)AE, (serious) adverse event; 5-ARI, $5 \alpha$-reductase inhibitors; AUA-SI, AUA Symptom Index; BII, $\mathrm{BPH}$ Impact Index; HR, hazard ratio; MTOPS, The Medical Therapy of Prostatic Symptoms (trial); NIDDK, the National Institute of Diabetes and Digestive and Kidney Diseases; PVR, post-void residual urine volume; $\mathrm{Q}_{\max }$, maximum urinary flow rate.

\section{Appendix 1}

Impact and symptom indices for BPH (adapted from Barry MJ. Evaluation of symptoms and quality of life in men with benign prostatic hyperplasia. Urology 2001; 58 (Suppl. 1): 25-32.)

Benign Prostatic Hyperplasia Impact Index (BPH-II)

\begin{tabular}{|c|c|c|c|c|c|}
\hline & None & Only a little & Some & A lot & \\
\hline 1. Over the past month, how much physical discomfort did any urinary problems cause you? & 0 & 1 & 2 & 3 & \\
\hline \multirow{2}{*}{$\begin{array}{l}\text { 2. Over the past month, how much did you worry about your health because of any urinary } \\
\text { problems? }\end{array}$} & 0 & 1 & 2 & 3 & \\
\hline & $\begin{array}{l}\text { Not at all } \\
\text { bothersome }\end{array}$ & $\begin{array}{l}\text { Bothers } \\
\text { me a little }\end{array}$ & $\begin{array}{l}\text { Bothers } \\
\text { me some }\end{array}$ & $\begin{array}{l}\text { Bothers } \\
\text { me a lot }\end{array}$ & \\
\hline \multirow[t]{2}{*}{ 3. Overall, how bothersome has any trouble with urination been during the past month? } & 0 & 1 & 2 & 3 & \\
\hline & $\begin{array}{l}\text { None of } \\
\text { the time }\end{array}$ & $\begin{array}{l}\text { A little of } \\
\text { the time }\end{array}$ & $\begin{array}{l}\text { Some of } \\
\text { the time }\end{array}$ & $\begin{array}{l}\text { Most of } \\
\text { the time }\end{array}$ & $\begin{array}{l}\text { All of } \\
\text { the time }\end{array}$ \\
\hline \multirow{2}{*}{$\begin{array}{l}\text { 4. Over the past month, how much of the time has any urinary problem kept you from doing } \\
\text { the kind of things you would usually do? }\end{array}$} & 0 & 1 & 2 & 3 & 4 \\
\hline & \multicolumn{5}{|c|}{ Total Score: } \\
\hline
\end{tabular}




\section{Appendix 2}

Full Model - Clinical decision tool for predicting 4-year risk of clinical BPH progression using data from MTOPS. In order to determine an individual's risk of progression, draw a vertical line from each of the patient's factors to the 'Points' line above. Add the six 'Points' values together to arrive at a 'Total Points'. Calculate the expected event rate with therapy by applying the hazard ratios below.

Points

Age, years

AUA Symptom Score

BPH Impact Index

PVR, mL

$\mathrm{Q}_{\max }, \mathrm{mL} / \mathrm{s}$

PSA, ng/mL
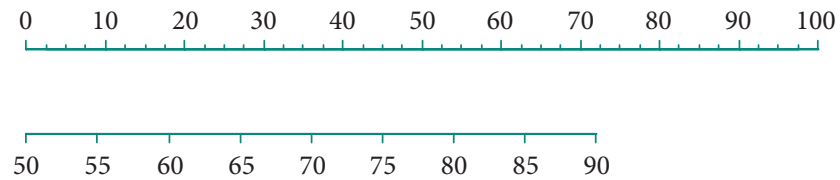

$\begin{array}{lllllllllllllll}36 & 34 & 32 & 30 & 28 & 26 & 24 & 22 & 20 & 18 & 16 & 14 & 12 & 10 & 8\end{array}$

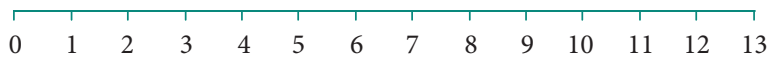

\begin{tabular}{lllllllll}
\hline 0 & 100 & 200 & 300 & 400 & 500 & 600 & 700 & 800
\end{tabular}

$\begin{array}{lllllll}16 & 14 & 12 & 10 & 8 & 6 & 4\end{array}$



\begin{tabular}{|c|c|c|}
\hline$<143$ & then risk quartile $=$ & $\begin{array}{l}158-181 \\
\text { artile }=\end{array}$ \\
\hline Q1 & Q2 & Q3 \\
\hline
\end{tabular}

4-year rate of progression without treatment

$10.70 \% \quad 12.80 \% \quad 18.90 \% \quad 29.60 \%$

To calculate event rate with therapy, multiple by treatment hazard ratio ( $95 \%$ confidence interval) Doxazosin Finasteride Combination $0 . \overline{54(0.40,0.72)} \quad 0.60(0.45,0.80) \quad 0.27(0.19,0.39)$

\begin{tabular}{|c|c|c|c|}
\hline \multicolumn{4}{|c|}{ if total points $=$} \\
\hline$<143$ & $143-158$ & $158-181$ & $>181$ \\
\hline Q1 & Q2 & Q3 & Q4 \\
\hline \multicolumn{4}{|c|}{ 4-year rate of progression without treatment } \\
\hline $10.70 \%$ & $12.80 \%$ & $18.90 \%$ & $29.60 \%$ \\
\hline
\end{tabular}

To calculate event rate with therapy, multiple by treatment hazard ratio ( $95 \%$ confidence interval) 